\section{§8. Characteristics of Honeycomb Catalysts for Oxidation of Tritiated Hydrogen and Methane Gases}

\author{
Uda, T., Tanaka, M.,
}

Munakata, K. (Interdisciplinary Graduate School of Engineering Sciences Kyushu University)

To oxidize the isotopic hydrogen and its compound gases to water form by catalyst is a conventional method for removal of tritium from air in a tritium processing facility. If tritium release accident occurs in a fusion facility, large volumes of air would be processed by the air cleanup system. However high throughput of air would cause pressure drop in catalyst beds, it results in high load to the process gas pumping system. In this study, applicability of honeycomb catalysts to the tritium recovery system was examined. The honeycomb catalyst bed has an advantage in terms of pressure drop, which is estimated to be less than $1 / 10$ of conventional particle-packed catalyst beds. Our previous studies revealed that honeycomb catalysts made of cordierite or Al-Cr-Fe metal alloy have preferable oxidizing performance. It was found that the platinum-deposited cordierite catalyst shows high oxidation rate for hydrogen gas, and the palladium-deposited metal honeycomb catalyst shows high oxidation rate for methane gas.

In this study, the properties of honeycomb catalysts were systematically studied by changing experimental parameters such as precious metal content, mesh density and so forth to obtain design data base for high performance honeycomb catalysts. As parameters the weighing rate of precious metal deposited on the honeycomb substances were varied from $1 \mathrm{~g} / \mathrm{L}$ to $4 \mathrm{~g} / \mathrm{L}$ and the mesh density of the honeycombs were changed from 260 to 400 CPSI as well. For operating conditions, the flow rate of the process gases, and the concentration of water vapor was varied.

Results of experimental study are as follows. The oxidation activity depends on weighing ratio of precious metal as shown in figure 1. But the mesh density CPSI has not been effective factor on oxidation. To avoid the pressure drop in the catalyst bed, lower CPSI would be preferable. The oxidation efficiency is large in small flow rate, namely small volume velocity. Of course large volume velocity is required in the catalytic oxidization process. In the next step, we examined affect of high moisture gas. Then oxidation efficiency for both hydrogen and methane gas decreases in case of including water vapor. Actually processing air by the cleanup system may be including some moisture element. So that the catalytic reactor should be designed by consider the loss of activity by moisture in air.

As conclusion, honeycomb catalysts are useful for the treatment of gases with high volumetric velocity in a fusion plant because of their low pressure drop in the catalyst reactor. The platinum catalysts were found to be suitable for oxidation of hydrogen gas, while the palladium catalysts exhibit better performance for oxidation of methane gas. With regard to hydrogen oxidization, the cordierite honeycomb reveals a better oxidizing performance than the metal alloy honeycomb. It was also suggested that the oxidization rate depends on the amount of deposited precious metal but the mesh density does not strongly affects the oxidation rate. Experimental results indicate that the catalytic oxidization rate decreases with increasing moisture content in the experimental gases. It was found that the catalytic activity of palladium catalysts for hydrogen oxidization is substantially decreased under the condition of low temperature and high humidity. In conclusion, it can be said that the honeycomb catalysts are promising alternatives of conventional packed bed type of catalyst for the recovery of tritiated gas if proper precious metal is selected. Although the cordierite honeycomb possesses better oxidizing property than the metal honeycomb, the metal honeycomb has an advantage for oxidation of hydrogen gas under room temperature, because it does not have structural water molecule that would cause memory effects via tritium contamination.

We also obtained design data base for conventional particle catalyst. Based on the database, the catalyst volume can be estimated for large volumetric velocity for example 1000 $\mathrm{Nm}^{3} /$ hour. It was found that these honeycomb catalysts can be used for the high-performance removal system of tritium from tritiated gases. These honeycomb catalyst are also applicable to the collection of tritiated gases for tritium measurement in atmospheric air around the fusion facility and the environment ${ }^{1)}$.

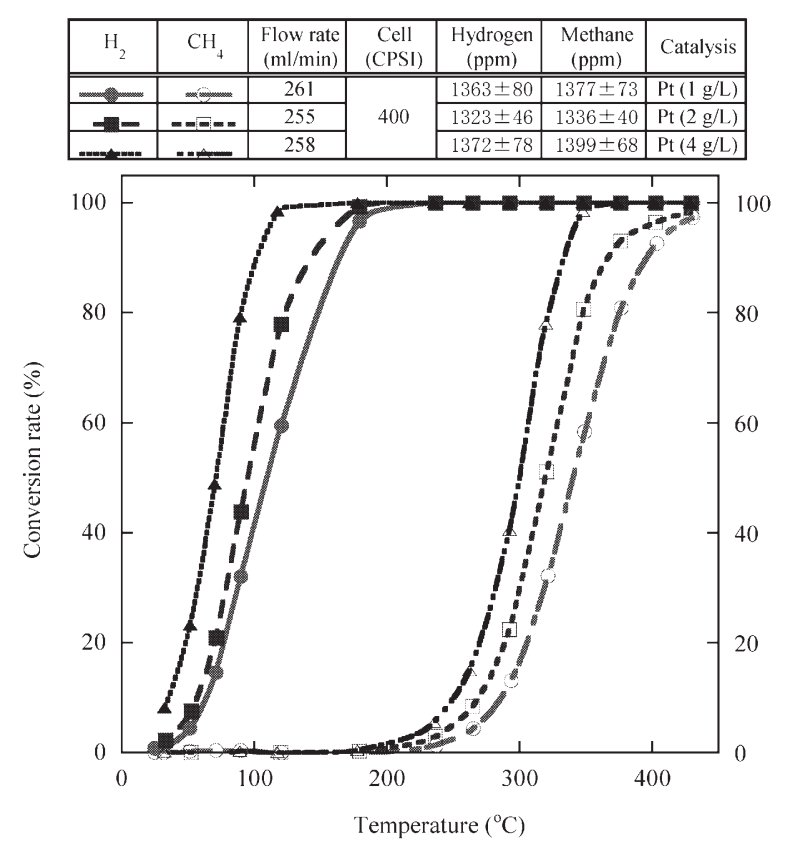

Fig. 1 Effect of precious metal weighing ratio on catalyst to oxidation.

1) . T. Uda, T. Sugiyama, K Munakata, M. Tanaka and N. Momoshima, "Developments of gaseous water, hydrogen and methane sampling system for environmental tritium monitor", Fusion Engineering and Design, 81 (2006) 1385. 